\title{
FAST FULLY AUTOMATIC BRAIN DETECTION IN FETAL MRI USING DENSE ROTATION INVARIANT IMAGE DESCRIPTORS
}

\author{
Bernhard Kainz ${ }^{1 *} \quad$ Kevin Keraudren ${ }^{1}$ \\ Joseph V. Hajnal ${ }^{2}$ \\ Vanessa Kyriakopoulou ${ }^{2}$ \\ Daniel Rueckert ${ }^{1}$ \\ Mary Rutherford ${ }^{2}$ \\ ${ }^{1}$ Biomedical Image Analysis Group, Department of Computing, Imperial College London, UK \\ ${ }^{2}$ Department Biomedical Engineering Division of Imaging Sciences, King's College London, UK
}

\begin{abstract}
Automatic detection of the fetal brain in Magnetic Resonance (MR) Images is especially difficult due to arbitrary orientation of the fetus and possible movements during the scan. In this paper, we propose a method to facilitate fully automatic brain voxel classification by means of rotation invariant volume descriptors. We calculate features for a set of 50 prenatal fast spin echo $\mathrm{T} 2$ volumes of the uterus and learn the appearance of the fetal brain in the feature space. We evaluate our novel classification method and show that we can localize the fetal brain with an accuracy of $100 \%$ and classify fetal brain voxels with an accuracy above $97 \%$. Furthermore, we show how the classification process can be used for a direct segmentation of the brain by simple refinement methods within the raw MR scan data leading to a final segmentation with a Dice score above 0.90 .
\end{abstract}

Index Terms - fetal MRI reconstruction, fetal brain localization, fetal brain segmentation

\section{INTRODUCTION}

Magnetic resonance imaging (MRI) of the fetus has been shown to be a useful tool for accurate prenatal diagnostics and to assess fetal development. Currently, mainly the brain and the whole fetus appearance are qualitatively examined in clinical practice $[1,2]$. Fetal motion and its unpredictable nature (Fig. 2) put high demand on radiologists and make an automatic evaluation of the scan challenging.

In this paper we propose rotation invariant volume descriptors [3] for medical imaging in combination with state-of-theart machine learning methods to provide a robust detection of the fetal brain. This detection process produces a reliable classification of brain voxels. It can simplify the application of advanced motion correction methods [4], which produce a high resolution volume from consecutive single-shot fast spin echo (SSFSE) T2-weighted scans. Alternatively, it can serve as a starting point for automatic or interactive segmentation. To demonstrate the feasibility of our method, we chose the latter application additionally to the localization challenge, and show that we can use the probability map resulting from the localization process for a basic brain segmentation.

* Bernhard Kainz is supported by a Marie Curie Intra-European Fellowship (FP7-PEOPLE-2012-IEF F.A.U.S.T. 325661).
Related work: Fetal MRI is a relatively new field, with little work published on fully automatic processing of these datasets. In [5, 6], 3D template matching is used to detect the eyes, enabling a subsequent 2D/3D graph-cut segmentation to extract the brain. This approach based on 3D rigid templates lacks the flexibility necessary to deal with motion artifacts as well as fetal malformations. The methods proposed in [7] and [8] address the variability of fetal MRI through machine learning. In [7], a Random Forest classifier first distinguishes between maternal and fetal tissues before identifying different tissues of the fetal head, while [8] combines prior knowledge of the fetal head size with MSER detection and a bag-of-words model. In contrast to [7], which obtains rotation invariance by rotating the training data and [8], which focuses on 2D slice detection, our method operates fully in 3D space, learning rotation invariant features, and is likely to be faster than all the methods proposed so far.

Contributions: To determine the orientation of the fetus, an automatic detection could try to register the scan to a known reference coordinate frame. This, however, is infeasible due to the influence of maternal tissues and the non-rigid nature of the fetus as a whole. A different approach is to derive image descriptors that are similar in any orientation of the image. Descriptors with such properties are called rotation invariant. We propose a novel way to apply rotation invariant feature descriptors based on Spherical Harmonics to medical volume images and show their potential to solve the orientation problem. The fetus is not sedated and may move during the volume acquisition, leading to motion artifacts between planes. By choosing the right trade-off between feature description accuracy and robustness of the descriptor, we can minimize the influence of motion artifacts on the detection process.

Fetuses develop rapidly and organ shapes and internal appearance change drastically over time. Our test dataset contains 50 fetuses aged between 22-37 weeks. Our brain classification method must be insensitive to local changes in appearance, which can be achieved by a correct choice of the feature descriptor parameters to average out small local details while preserving the general appearance in feature space. By simple post-processing of the detection probability map, we also show how to obtain a basic segmentation of the fetal brain. 


\section{METHOD}

Fig. 1 provides an overview of our brain detection pipeline.

\begin{tabular}{|c|c|c|c|c|}
\hline $\begin{array}{l}\text { Labeled } \\
\text { Scan 1...n }\end{array}$ & $\begin{array}{l}\text { Normalize and } \\
\text { NLM denoising }\end{array}$ & $\begin{array}{l}\text { Feature-space } \\
\text { transformation }\end{array}$ & $\begin{array}{c}\text { Combine feature } \\
\text { vector }\end{array}$ & $\begin{array}{l}\text { Train Regression } \\
\text { Forest classifier }\end{array}$ \\
\hline & & & & 7 \\
\hline $\begin{array}{l}\text { Test } \\
\text { scan }\end{array}$ & $\begin{array}{l}\text { Normalize and } \\
\text { NLM denoising }\end{array}$ & $\begin{array}{l}\text { Feature-space } \\
\text { transformation }\end{array}$ & $\begin{array}{l}\text { Predict with } \\
\text { classifier 3D }\end{array}$ & $\begin{array}{l}2 \mathrm{D} \text { region } \\
\text { refinement }\end{array}$ \\
\hline
\end{tabular}

Fig. 1. Method overview: after normalization and non-local median (NLM) denoising, each training image is transformed to feature space and feature vectors are learned by a Random Forest. The resulting classifier is used to detect the brain from a new dataset, its probability map providing a rough initial segmentation which is later used in a $2 \mathrm{D}$ refinement.

Image acquisition: Images are acquired on a Philips Achieva $1.5 \mathrm{~T}$ scanner, the mother lying either on the back or on the side in order to feel comfortable. A typical acquisition begins with a localizer scan, which is used to align the scan main axis approximately parallel to the fetus. Single-shot fast spin echo (SSFSE) T2-weighted sequences are used to acquire a stack of images of the mother's womb. Each acquisition of a 2D image takes approximately $0.5-1.0 \mathrm{~s}$, which makes throughplane movement artifacts very likely until the whole image stack is available. Several of these image stacks are usually acquired, parallel and perpendicular to the fetus' main axis. This allows to account for movement artifacts during later post-processing steps [4]. In this work, we use one randomly selected image stack from each subject to show our method's ability to cope with through-plane movement artifacts. Example slices from our datasets are shown in Fig. 2. All fetal subjects have gestational ages between 22-37 weeks.

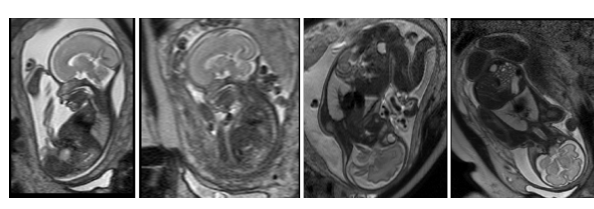

(a)

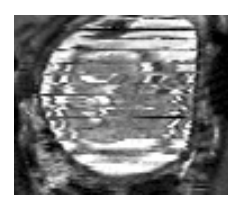

(b)
Fig. 2. Example slices from our training data: (a) exemplary slices: The fetus can be randomly oriented. The throughplane resolution is low and moving artifacts can occur during the acquisition of the slices as shown in the multi-planar reconstruction (MPR) in (b).

Transformation to a rotation invariant feature space: Before transforming the image to feature space, we we normalize the image intensities and apply a non-local median denoising filter [9]. We define rotation invariant features with the angular power spectrum $\left\|a^{l l}\right\|^{2}$ of the expansion coefficients $a^{l}$ of Spherical Harmonic Functions similar to Skibbe et al. [3]. Skibbe et al. evaluate several different basis functions and show strong evidence that the power spectrum of the expansion with spherical 3D Gabor basis functions, represented by a superposition of Bessel functions $\mathcal{B}_{s}^{l}(k)$, are highly suitable for both classification accuracy and computation time when used for medical 3D images. Thus, we also define our spherical 3D Gabor descriptors (SGD) coefficients at an expansion $l$, scale $t$ and frequency $k$ with $a_{l}^{k}(x)=\left(\frac{\sqrt{t}}{-k}\right)^{l} \bar{\nabla}^{l}\left(I \star \mathcal{B}_{s}^{0}(k)\right)(x)$. The transformation into the Gabor domain can be realized with one single initial cross correlation of the image and the basis function $I \star \mathcal{B}_{s}^{0}(k)$ followed by an iterative application of the spherical up-derivative operator $\bar{\nabla}^{l}$, which defines the spherical counterpart of a gradient operator in conventional calculus [3]. A major difference between the work by Skibbe $e t$ al. and ours is that our feature vectors do not describe structures as a whole but rather small patches of the organ. We also implemented our own version based on Nvidia CUDA [10] and accelerated the algorithm to achieve an approximately 60 times faster feature space transformation.

Furthermore, we determined the best trade-off between SGD detail and computation performance using cross-validation on two randomly selected datasets that have been excluded from the training and testing datasets. We found the optimal expansion bandwidth to be around 20 derivatives.

Cross-validation was also used to find the best size of the voxel set covered by one descriptor. In contrast to Skibbe $e t$ $a l$., we do not describe the whole object (organ) in harmonics space, but a small subset of the organ at every position in the volume. We obtained the best results with descriptor sizes between $3-5 \mathrm{~mm}$. We combine features at the minimum and the maximum of this range to form a feature vector consisting of coefficients for a smaller and a larger spherical neighborhood to gain additional descriptive power. Our final SGD covers three different frequencies to represent the local image structure at each scale. We use $k=0, \pi$, and $2 \pi$. Therefore, each SGD feature descriptor vector has a length of 66 elements.

Learning of brain features: To improve learning performance and maintain a spherical shape of the descriptor in the feature space, the data is subsampled in-plane to match the through-plane resolution, so that the input image has an isotropic voxel size.

We train a state-of-the-art Classification Forest ensemble learning method based on decision trees for the SGD image features [11,12], because of its trade-off between efficiency and classification performance. All descriptors labeled as brain and a third of the background descriptors to reduce training time are used for learning.

To tune the decision tree preference toward the brain features, the weights of misclassified brain voxels are altered according to the average ratio of the size of the fetal brain compared to the 3D volume of the whole scan. In our datasets, approximately $10 \%$ of the voxels belong to the brain and $90 \%$ belong to background. A maximum of 256 trees have been used, with a maximum depth of 256. During cross-validation, these values resulted in the best trade-off between computation time and classification performance. An example probability map 
of the prediction for a test-image is presented in Fig. 3(a). Using the largest region with the highest probabilities $(p>0.8)$ results in a highly accurate area within the fetal brain.

Fetal brain segmentation refinement: To demonstrate the feasibility of our method to serve as a basis for further postprocessing, we use the probability map from the Classification Forest as input for a slice based brain segmentation. We use 2D slices parallel to the two smallest voxel sides for this example because of the large through-plane resolution of our datasets $(4 \mathrm{~mm})$ and the small volume of the fetus.

The probability map is first thresholded at a value higher than 0.5 . While outlying regions may have probabilities above 0.5 , the values for true brain voxels are generally higher. To remove possible remaining outlying regions, the largest 3D connected component is selected. During our experiments, this approach always resulted in an area within the fetal brain (localization accuracy $=100 \%$ ). For each 2D slice, the minimum ( $\min$ ) and maximum ( $\max$ ) intensity values of the intersection of the thresholded probability map with the slice is computed. Taking advantage of the bright appearance of brain pixels in T2 MRI, all pixels on a slice whose intensity value is smaller than $\min -0.1 \cdot(\max -\min )$ are set to the minimum of the whole volume.

Finally, a 2D level-set [13] is initialized from the thresholded probability map and evolved on the processed slices. A few hundred iterations are usually sufficient for an accurate segmentation of the fetal brain.

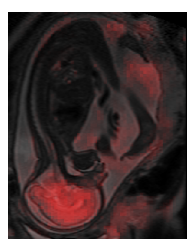

(a)

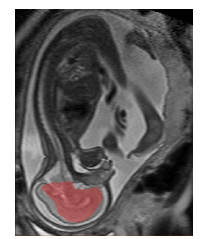

(b)

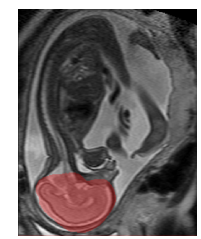

(c)

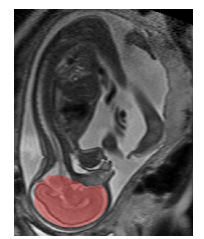

(d)
Fig. 3. The probability map for a test dataset is colored red the higher the probability for a brain voxel is (a). (b) shows the detection result, which is the input for the segmentation refinement process after thresholding the probability map and selection of the largest connected region. (c) shows the final result after simple refinement, and (d) shows the ground truth expert segmentation. We use [14] for visualization.

\section{RESULTS}

We have tested our method on 50 datasets semi-automatically segmented using manually initialized graph cuts. The datasets have been cropped to an average size of $200 \times 200 \times 70$ voxels encompassing the whole fetal body in order to reduce the number of background voxels and therefore the computation time during testing. Note that our method shows the same performance without cropping but at higher computational costs. We have randomly picked ten times ten different datasets as test set and learned the appearance of the brain in the feature space from the remaining 40 datasets. Tab. 1 summarizes the averaged results for the detection process, considering values above 0.5 in the probability map as correct classification. The probability in the center regions of the brain is usually $>0.85$, which can be taken as threshold value if localization is the target application. During our experiments, the centroid of the largest area classified with a brain probability above 0.85 showed a $100 \%$ localization accuracy.

Using a probability threshold above 0.5 reaches a classification accuracy of over $97 \%$ on average without refinement. However, since most of the voxels in a dataset represent background information, a method predicting always zero would also have a high accuracy. Therefore, we also evaluated the weighted average of Precision and Recall with the DICE coefficient (DC) in Tab. 1. An average DC of $0.850 \%$ before segmentation refinement shows that our base method is already highly robust and that it shows only a few to no outliers.

We evaluate the refined brain segmentation according to [15] in Tab. 1. Considering the low through-plane resolution of our datasets, the average distance values show that we can reliably segment the brain in most slices. The maximum distance errors show that our refinement method has limitations in regions with large anatomical abnormalities, where an unusually large gradient will stop the level set evolution, and for slices where only a few voxels were detected as brain (border slices). The level set over-segments in these peripheral slices because of the absence of a clear gradient as well as partial volume effects from the skull. It produces a region which is on average $5-10 \mathrm{~mm}$ too large. These border slices are mainly responsible for higher average and maximum surface distance errors and could be left out in practice.

Figure 3 shows an example result from our test-dataset. Our method has been evaluated on an Intel Xeon E5-2643 system with 16 GB RAM and an Nvidia Titan Graphics card. The transformation to feature space (GPU) and voxel classification (CPU) takes on average $1.4 \mathrm{~s}$. The segmentation refinement (CPU) takes another $5.7 \mathrm{~s}$. Therefore, our approach can be executed within approximately $7 \mathbf{s}$.

Note that our method provides the same $100 \%$ localization accuracy as [8]. However, our approach is faster to compute and provides additionally an accurate brain segmentation mask.

\section{CONCLUSION AND FUTURE WORK}

We have presented a fast, reliable and fully automatic way to localize the fetal brain from prenatal volumetric MRI scans. Our approach allows the automatic classification of a set of voxels within the fetal brain with high precision and recall. While previous methods were limited to the localization of the brain, we also show that our detection process can serve as a suitable prior for further fully automatic segmentation of the fetal brain. Our method is fast enough for a near real-time application to the scan during the acquisition process and can provide essential input for subsequent scanning procedures. 


\begin{tabular}{|c|c|c|c|c|c|c|c|c|c|}
\hline \multirow[b]{2}{*}{ Experiment } & \multicolumn{4}{|c|}{ Voxel classification } & \multicolumn{5}{|c|}{ Segmentation refinement } \\
\hline & Accuracy & Precision & Recall & DC & RAVD & ASSD & RMSSSD & MSSD & $\mathrm{DC}$ \\
\hline 1 & 0.98 & 0.87 & 0.84 & 0.85 & 2.91 & 4.21 & 11.42 & 21.42 & 0.89 \\
\hline 2 & 0.97 & 0.88 & 0.82 & 0.85 & 2.64 & 4.15 & 10.53 & 24.53 & 0.91 \\
\hline 3 & 0.96 & 0.83 & 0.81 & 0.82 & 2.73 & 4.52 & 9.31 & 19.52 & 0.92 \\
\hline 4 & 0.97 & 0.89 & 0.82 & 0.85 & 3.31 & 5.03 & 11.42 & 28.42 & 0.89 \\
\hline 5 & 0.98 & 0.90 & 0.83 & 0.86 & 2.84 & 4.30 & 10.49 & 23.04 & 0.89 \\
\hline 6 & 0.95 & 0.84 & 0.82 & 0.83 & 2.99 & 4.24 & 12.82 & 22.75 & 0.90 \\
\hline 7 & 0.98 & 0.87 & 0.86 & 0.87 & 2.61 & 4.15 & 11.99 & 20.42 & 0.91 \\
\hline 8 & 0.97 & 0.88 & 0.86 & 0.87 & 2.31 & 4.17 & 10.42 & 17.53 & 0.92 \\
\hline 9 & 0.98 & 0.87 & 0.86 & 0.87 & 2.44 & 4.21 & 9.62 & 20.21 & 0.93 \\
\hline 10 & 0.98 & 0.86 & 0.83 & 0.84 & 2.95 & 4.33 & 11.83 & 22.01 & 0.91 \\
\hline Average & 0.971 & 0.868 & 0.833 & 0.850 & 2.773 & 4.331 & 10.985 & 21.985 & 0.907 \\
\hline
\end{tabular}

Table 1. Voxel classification, left: Performance results for the brain classification method. These measures reflect the classifier performance without any post-processing. Using the simplest refinement method from Section 2 - selecting the largest connected region - would lead to a mean DC $>0.88$. Segmentation refinement, right: Results from our segmentation refinement process for our validation experiment. The table shows measures according to [15]: relative absolute volume difference (RAVD), average symmetric surface distance (ASSD), root mean square symmetric surface distance (RMSSSD), maximum symmetric surface distance (MSSD), and average Dice coefficient (DC).

http://goo.gl/uKNgXf shows a video about our method.

\section{REFERENCES}

[1] M. Rutherford, S. Jiang, J. Allsop, L. Perkins, L. Srinivasan, T. Hayat, S. Kumar, and J. Hajnal, "MR imaging methods for assessing fetal brain development," Dev Neurobiol, vol. 68, no. 6, pp. 700-11, 2008.

[2] C. Limperopoulos and C. Clouchoux, "Advancing fetal brain MRI: targets for the future," Seminars in perinatology, vol. 33, no. 4, pp. 289-298, Aug. 2009.

[3] H. Skibbe, M. Reisert, T. Schmidt, T. Brox, O. Ronneberger, and H. Burkhardt, "Fast Rotation Invariant 3D Feature Computation utilizing Efficient Local Neighborhood Operators," IEEE Transactions on Pattern Analysis and Machine Intelligence, vol. 34, no. 8, pp. 1563 1575, Aug 2012.

[4] M. Kuklisova-Murgasova, G. Quaghebeur, M. A. Rutherford, J. V. Hajnal, and J. A. Schnabel, "Reconstruction of fetal brain MRI with intensity matching and complete outlier removal," Elsevier Medical Image Analysis, vol. 16, no. 8, pp. 1550 - 1564, 2012.

[5] J. Anquez, E. D. Angelini, and I. Bloch, "Automatic segmentation of head structures on fetal MRI," in in Proc. IEEE International Symposium on Biomedical Imaging. 2009, pp. 109-112, IEEE Press.

[6] Y. Taleb, M. Schweitzer, C. Studholme, M. Koob, J.L. Dietemann, and F. Rousseau, "Automatic Templatebased Brain Extraction in Fetal MR Images," Abstract hal-00879089, 62013.

[7] M. Ison, R. Donner, E. Dittrich, G. Kasprian, D. Prayer, and G. Langs, "Fully Automated Brain Extraction and Orientation in Raw Fetal MRI," in Proc. of MICCAI Workshop on Paediatric and Perinatal Imaging, 2012.

[8] K. Keraudren, V. Kyriakopoulou, M. Rutherford, J.V. Hajnal, and D. Rueckert, "Localisation of the Brain in Fetal MRI Using Bundled SIFT Features," in MICCAI 2013, vol. 8149 of LNCS, pp. 582-589. 2013.

[9] Y. Li and S. Osher, "A new median formula with applications to PDE based denoising," Commun. Math. Sci., vol. 7, no. 3, pp. 741-753, 2009.

[10] NVIDIA Corporation, NVIDIA CUDA Compute Unified Device Architecture Programming Guide, NVIDIA Corporation, 2012.

[11] A. Criminisi and J. Shotton, Decision Forests for Computer Vision and Medical Image Analysis, SpringerVerlag New York Incorporated, 2013.

[12] G. Bradski, "The OpenCV Library,” Dr. Dobb's Journal of Software Tools, 2000.

[13] R. Malladi, J.A. Sethian, and B.C. Vemuri, "Shape Modeling with Front Propagation: a Level Set Approach," IEEE Transactions on Pattern Analysis and Machine Intelligence, vol. 17, no. 2, pp. 158-175, 1995.

[14] I. Wolf, M. Vetter, I. Wegner, T. Böttger, M. Nolden, M. Schöbinger, M. Hastenteufel, T. Kunert, and H.-P. Meinzer, "The Medical Imaging Interaction Toolkit.," Elsevier Medical Image Analysis, vol. 9, no. 6, pp. 594604, 2005.

[15] B. Van Ginneken, T. Heimann, and M. Styner, "3D segmentation in the clinic: A grand challenge," in In: MICCAI Workshop on 3D Segmentation in the Clinic: A Grand Challenge., 2007. 